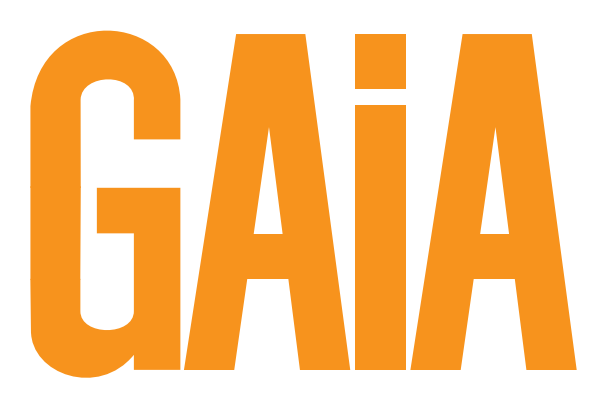

$2 \mid 2016 \begin{aligned} & \text { ECOLOGICAL PERSPECTIVES FOR SCIENCE AND SOCIETY } \\ & \text { ÖKOLOGISCHE PERSPEKTIVEN FÜR WISSENSCHAFT UND GESELLSCHAFT }\end{aligned}$

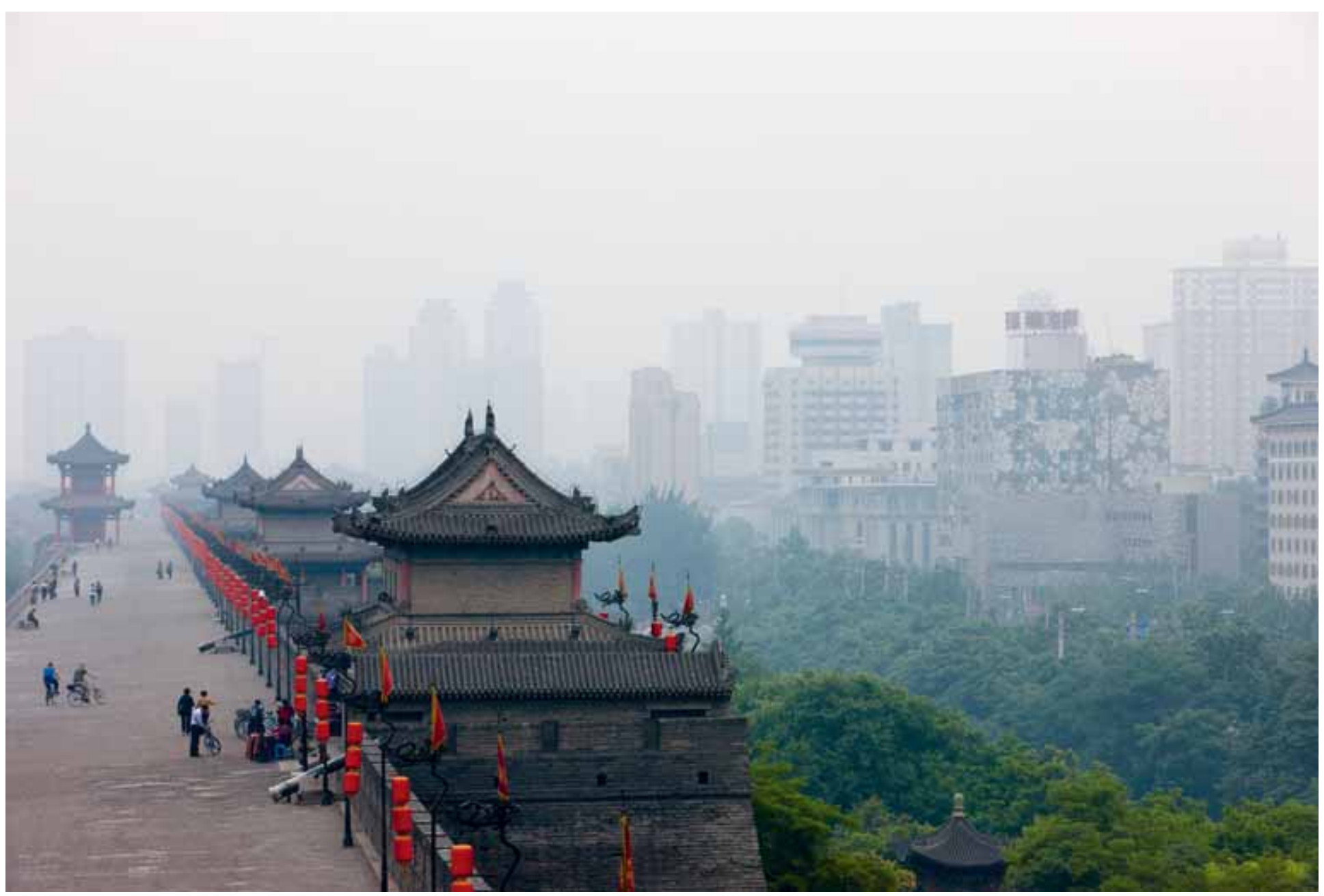

FORSCHUNG FÜR UND ÜBER TRANSFORMATION

- FRACKING UND LANDSCHAFT

- NACHHALTIGKEIT ALS FORM DES NIESSBRAUCHS 


\title{
Verdichtung: diskursanalytisch beleuchtet
}

\author{
Reaktion auf I. Krau in GAIA 25/2 (2016, in diesem Heft): \\ Ein Wort geht um: Verdichtung
}

Hugo Caviola, Andreas Kläy, Hans Weiss

Densification from the Perspective of Discourse Analysis | GAIA 25/2 (2016): 86-87

Keywords: cognitive metaphor theory, discourse analysis, sustainable urban development, urban densification, urban ecology

$D^{\prime}$ e Reaktion von Ingrid Krau (2016, in diesem Heft) ist dankenswert, da sie unsere Thematik aus städtebaulicher Sicht vertieft. Im Folgenden gehen wir auf vier Aspekte ein, in denen uns die Verfasserin offenbar missverstanden hat.

\section{Multiperspektivität}

Es ist tatsächlich so, dass unser Autorenteam nicht aus den Planungswissenschaften stammt und daher nicht aus dem entsprechenden Insiderwissen heraus argumentiert. Dies mag da und dort zu Unschärfen und Auslassungen geführt haben. Mit unserem sprachanalytischen Zugang wählten wir aber ausdrücklich einen Blickwinkel, der von außen auf die Begriffe schaut. Denn mit demselben Recht, mit dem man uns hier aus Sicht der Planungswissenschaften kritisiert, könnten auch Vertreter(innen) der Soziologie, der Psychologie, der Biologie, der Rechtswissenschaften, der Ökonomie oder der Geschichtswissenschaften auftreten und monieren, dass ihre Sichtweisen auf den Siedlungsbau zu kurz gekommen sind.

Unser Zugang - wie wir eingangs klarmachen - ist ein diskursanalytischer. Daraus folgt, um es noch einmal zu betonen, dass wir ganz bewusst eine reflektierende Außensicht einnehmen, aus der wir sprachlich vermittelte Perspektiven sichtbar machen wollen. Perspektiven sind nach unserem Verständnis nicht zwingend fachlich gebunden. So lässt sich städtischer Siedlungsbau aus ver-

Kontakt: Dr. Hugo Caviola | Tel.: +41 619212171 |

E-Mail: hugo.caviola@cde.unibe.ch

Andreas Kläy, Ing. ETH | E-Mail: andreas.klaey@cde.unibe.ch

beide: Universität Bern | Interdisziplinäres Zentrum für Nachhaltige Entwicklung und Umwelt (CDE) | Hallerstr. 10 | 3012 Bern | Schweiz

Hans Weiss, Kulturingenieur ETH | Bern | Schweiz | E-Mail: hweiss@bluewin.ch

(C) 2016 H. Caviola et al.; licensee oekom verlag. This is an article distributed under the terms of the Creative Commons Attribution License (http://creativecommons.org/licenses/by/3.0), which permits unrestricted use, distribution, and reproduction in any medium, provided the original work is properly cited. schiedenen Sichtweisen erschließen: jener der Stadtplanung, der Architektur, der Banken, der Bauarbeiter(innen), aber auch der Bewohner(innen), bis hin zu den Perspektiven, die man Tieren und Pflanzen zuschreiben kann. Sprachliche Perspektiven sind durch sogenannte Deutungsrahmen (Frames) bestimmt, die oft über konzeptuelle Leitmetaphern vermittelt werden. Unsere Untersuchung kommt zu dem Ergebnis, dass innerhalb der untersuchten Texte zum Siedlungsbau zwei Leitmetaphern vorkommen: eine dominante, die physikalische, und eine sekundäre, die Entwicklungsmetapher (zum Beispiel in Stadtentwicklung, Siedlungsentwicklung). Das Ziel unseres Artikels ist erstens, diese Perspektivierungen und die mit ihnen vermittelten Interessen bewusst zu machen. Zweitens plädieren wir im Sinne eines multiperspektivischen Zugangs dafür, die Entwicklungsperspektive zu stärken. Dies kann zum Beispiel durch die Leitmetapher „die Siedlung ist ein Organismus“ geschehen. Denn aus dieser Optik lassen sich die Lebensansprüche des verdichteten Menschen und der Pflanzen und Tiere sprachlich besser repräsentieren als durch den physikalischen Deutungsrahmen.

\section{Sprachverständnis}

Mit unserem diskursanalytischen Zugang betreten wir Neuland in der Diskussion über verdichtetes Bauen. Wir sind uns bewusst, dass das damit vertretene Sprachverständnis ein ungewohntes sein kann. Sprache, Denken und Handeln gehen für uns Hand in Hand. Was heißt das? Die Kritikerin erklärt, dass Planende, zum Beispiel Architekt(inn)en, in ihrer Ausbildung in einen geometrisch-mathematischen Umgang mit Baukörpern eingeübt werden. Dies ist zweifellos so. In dieser Ausbildung wird eine wichtige Grundlage für das physikalische Denken gelegt. (Dieses hat in der Baukunst auch seine Berechtigung, weil Baukörper unter anderem den Gesetzen der Statik zu gehorchen haben). Die Kommentatorin macht zu Recht auf diese fachliche Prägung des Architektenberufs aufmerksam. Aus diskursanalytischer Sicht geschieht jedoch eine solche Denkschulung durch Geometrie und 
Mathematik nicht außerhalb der Sprache. Entscheidend ist, dass geometrisch-mathematisches Wissen erstens an Fachbegriffe wie Fläche, Raum, Volumen, Baukörper gebunden ist und zweitens an ein mathematisches Kalkül (zum Beispiel der Subtraktion oder Division), das im Ganzen auch sprachgebunden ist.

\section{Methode}

Die Kommentatorin bezeichnet unsere Methode der Ermittlung der physikalischen Begriffe als schwer nachvollziehbar und erklärt, wir ließen neben den physikalischen ,andere Aspekte mit Leitcharakter“ außer Acht (Krau 2016, S. 84, in diesem Heft).

Es ist richtig, dass die von uns untersuchten Texte nicht allein von physikalischen Begriffen geprägt sind, sondern durchaus auch Einsprengsel aus anderen Begriffsfeldern wie zum Beispiel der Entwicklungsmetapher enthalten. Entscheidend ist aber, dass die Begriffe aus dem physikalischen Begriffsfeld nicht vereinzelt auftreten, sondern einen netzartigen Wissensrahmen bilden, innerhalb dessen diese Begriffe eine Denklogik entfalten, in der sie sich gegenseitig stützen und so in ihrer Plausibilität bestärken. Beispiel: Erhöhen wir in einem Siedlungsgebiet die Anzahl der Teilchen (Wohnungen/Menschen), so steigt die Teilchendichte und ein irgendwie gearteter Druck wird erhöht. In einer Entsprechungsmatrix haben wir in unserem Beitrag Begriffe aus dem Handlungsfeld verdichtetes Bauen den zentralen Dichtebegriffen der Physik zugeordnet (Caviola et al. 2016, S. 51, Tabelle 1). Die dort dargebotene Tabelle umfasst also nicht beliebige Begriffe, sondern einen in sich kohärenten Wissensrahmen, welcher der Physik angehört. Die „Beschränkung“ des untersuchten Begriffsfeldes, die moniert wird, betrifft wohl unseren ersten Arbeitsschritt: Wir suchen Fundstücke, welche unsere Hypothese untermauern. Im zweiten Arbeitsschritt aber öffnen wir das Visier und stellen andere Begriffe und Metaphern aus den Texten vor (Caviola et al. 2016, S. 54, Tabelle 2). Aus dem Miteinander der unterschiedlichen Deutungsrahmen ziehen wir am Ende unsere Schlüsse.

\section{Funktion der Bilder}

Missverstanden hat die Kommentatorin offensichtlich auch die Bedeutung unserer Illustrationen. Mit unseren Abbildungen 2 und 4 (abgedruckt als Abbildung 1 und 2 bei Krau 2016, S. 85, in diesem Heft) wollen wir keine konkreten Handlungsempfehlungen geben. Es geht uns vielmehr in beiden Fällen um die Illustration des Prinzips, wie menschliches Denken, Sprechen und Handeln zusammenhängen. So interessiert uns etwa am abgebildeten Zwischenraum (vergleiche Abbildung 1 in Krau 2016, S. 85, in diesem Heft) nicht, in welcher Weise hier baulich interveniert werden soll. Der stark geometrisierte Raum soll vielmehr einen Raumtyp darstellen, den man typischerweise als Bauabstand, Leerraum, Zwischenraum oder als Freiflüche anspricht. Die Kommentatorin schließt sich offensichtlich dieser Einschätzung an, wenn sie in ihrer Replik von einer Freiflächengestaltung schreibt (Krau 2016, S. 85, in diesem Heft). Entscheidend ist aus unserer Sicht, dass mit den genannten Ausdrücken sprachliche Perspektivierungen gewählt sind, die den benannten Raum aus der Sicht baulicher Interessen erschließen. Im Kontext des Bauens sind Freiflächen, Leerräume oder Zwischenräume von Baukörpern ausgenommene Räume. Wir wollen damit aufzeigen, dass andere als bauliche Interessen in diesen Formulierungen ausgeblendet werden oder zumindest sprachlich nicht direkt repräsentiert sind. Die Herausforderung verdichtenden Städtebaus liegt unserer Ansicht nach darin, neben den baulichen auch die sozialen und ökologischen Lebensansprüche sprachlich direkt zu repräsentieren.

Dass dies möglich ist, zeigt Frau Krau in ihrem Kommentar zum Projekt wagnisART auf, wenn sie von „großzügigen Gemeinschaftsflächen“ schreibt (Krau 2016, S. 85, in diesem Heft). Mit Gemeinschaftsflächen ist eine sprachliche Perspektivierung gewählt, die städtischen Lebensraum nicht als unüberbaut darstellt, sondern direkt aus Sicht der Bewohner(innen) erschließt und damit auch deren Interessen zum Ausdruck bringt. Interessant scheint uns am Modell von wagnisART auch, dass es uns sprachlich in soziale und ökologische Räume (aus früheren Zeiten) führt, wenn zum Beispiel von Wäldchen, Hain und Kirschengruß einerseits und von Dorfplatz, Marktplatz und Oasenhof andererseits die Rede ist (Krau 2016, S. 85, Abbildung 3, in diesem Heft).

\section{Fazit}

Wir wollen und können mit unserem Zugang nicht auf alle Herausforderungen des zeitgenössischen Siedlungsbaus eingehen. Unser Forschungsprojekt Sprachkompass Landschaft und Umwelt will allgemein das Sprachbewusstsein und damit das Reflexionswissen im Umgang mit Landschaft und Umwelt stärken. Wir untersuchen Begriffe, die im Umgang mit Landschaft zentral sind. Zu ihnen gehören etwa Raum, Fläche, Vernetzung, Landschaftsleistung, Entwicklung und Verdichtung. Wir fragen aus diskursanalytischer Sicht, was diese Begriffe sichtbar machen, was sie ausblenden und welche Interessen sie zum Ausdruck bringen. Unsere Website ${ }^{1}$ bietet Einblicke in unsere Arbeiten und lädt Interessierte zum Mitdenken und Mitwirken ein.

\section{Literatur}

Caviola, H., A. Kläy, H. Weiss. 2016. Im physikalischen Verdichtungslabor Wie Sprache das Denken und Handeln im Siedlungsbau beeinflusst. GAIA 25/1: 49-56.

Krau, I. 2016. Ein Wort geht um: Verdichtung. GAIA 25/2: 84-85.

1 www.sprachkompass.ch

Autorenbiografien siehe letztes Heft, GAIA 25/1 (2016): 56 